Editorial

\title{
Acknowledgement to Reviewers of Cosmetics in 2019
}

\section{Cosmetics Editorial Office}

MDPI, St. Alban-Anlage 66, 4052 Basel, Switzerland

Published: 19 January 2020

The editorial team greatly appreciates the reviewers who have dedicated their considerable time and expertise to the journal's rigorous editorial process over the past 12 months, regardless of whether the papers are finally published or not. In 2019, a total of 69 papers were published in the journal, with a median time to first decision of 13 days and a median time from submission to publication of 36 days. The editors would like to express their sincere gratitude to the following reviewers for their generous contribution in 2019:

\author{
Al-Fatimi, Mohamed \\ Alfonso, José \\ Amaral, Helena \\ Antoniotti, Sylvain \\ Apone, Fabio \\ Arsene, Andreea Letiţia \\ Badea, Nicoleta \\ Bagnato, Vanderlei Salvador \\ Baki, Gabriella \\ Barba, Clara \\ Basketter, David \\ Baswan, Sudhir \\ Beier, Ross \\ Berardesca, Enzo \\ Bogdan, Catalina \\ Brettell, Thomas \\ Calorini, Lido \\ Celeiro, María \\ Cernava, Tomislav \\ Cha, Hwa Jun \\ Chamcheu, Jean Christopher \\ Chang, Te-Sheng \\ Choi, Duhyung \\ Cidade, Maria Teresa \\ Coutinho, Paula \\ D'Amora, Ugo \\ De Paula, Haroldo César Beserra \\ De Totero, Daniela \\ Delgado-Charro, M. Begoña \\ Evans, Trefor A. \\ Ezendam, Janine \\ Feliciani, Claudio \\ Fölster-Holst, Regina \\ Fuller, Bryan \\ Fung, Ernest S.
}

\author{
Georgiev, Vasil \\ Goel, Peeyush N. \\ Goenka, Shilpi \\ González-Minero, Francisco José \\ Guzmán, Eduardo \\ Hall, Eric S. \\ Hara, Koji \\ Holefors, Anna \\ Hussain, Zahir M. \\ Ishihara, Kenji \\ Ita, Kevin \\ Ito, Takamichi \\ Jakasa, Ivone \\ Jeong, Se Kyoo \\ Jones, Alex \\ Jones, Alex L. \\ Juliano, Claudia Clelia Assunta \\ Kageyama, Hakuto \\ Kendall, Alexandra \\ Kim, Donguk \\ Koch, Sandra \\ Koike, Kenzo \\ Kojima, Hajime \\ Kolbe, Ludger \\ Krajka-Kuźniak, Violetta \\ Labrou, Nikolaos \\ Lagouri, Vasiliki \\ Lainé, Eric \\ Le Tilly, Véronique \\ Leijs, Marike \\ Lempart, Anna \\ Lin, Chih-Chien \\ Lionetti, Nicola \\ Lodyga-Chruscinska, Elżbieta \\ Lores, Marta
}


Madany Mamlouk, Amir

Maeda, Kazuhisa

Malinauskiene, Laura

Mallela, Shamroop Kumar

Manfredini, Stefano

Mäntele, Werner

Maria Lisa, Garavaglia

Marrelli, Mariangela

Marsh, Jennifer

Matamá, Maria Teresa

Matsugo, Seiichi

Matsunami, Katsuyoshi

Melucci, Dora

Menaa, Farid

Merah, Othmane

Mesnard, François

Mitjans Arnal, Montserrat

Mochizuki, Shinichi

Mokrejš, Pavel

Moldovan, Mirela

Nakatsuji, Teruaki

Napolitano, Alessandra

Nimse, Satish Balasaheb

Noda, Shinji

Nowicki, Roman

Panzella, Lucia

Park, Kyoung-Chan

Pereira, Leonel

Pérez, María del Carmen

Perugini, Paola

Petropoulos, Spyridon

Piccirillo, Clara

Pilmane, Māra

Plonka, Przemyslaw

Puviani, Mario

Qasim, Saad

Qassem, Meha

Quan, Taihao

Ribeiro, Artur

Rigano, Luigi

Rinnerthaler, Mark

Ripoll, Lionel

Roberts, David

Robinson, Deanne
Rodrigues, Francisca

Rogers, George Ernest

Roohnikan, Mahdi

Rua, Diego

Santos, Ana

Santos, Jenifer

Schleusener, Johannes

Semenzato, Alessandra

Serfaty, Stéphane

Sethi, Gautam

Shim, Sehwan

Shimo, Tsuyoshi

Shin, Hyun-Jae

Sikorska, Wanda

Silva, Carla

Simoes, Sandra

Singh, Anil

Sinha, Sudarson Sekhar

Sironi, Maurizio

Sitarek, Przemysław

Solano, Francisco

Sorg, Olivier

Sowa, Ireneusz

Staderini, Edoardo

Starace, Michela

Sugumaran, Manickam

Surber, Christian

Tanabe, Shihori

Thomas-Danguin, Thierry

Tobin, Desmond

Tobin, Desmond J.

Tolidis, Kosmas

Tóth, Gergő

Van Erp, Piet

Vassallo, Antonio

Verardo, Vito

Vincenzi, Colombina

Wang, Zhenping

Wondrak, Georg

Xiao, Perry

Yang, Chao-Hsun

Youssefian, Leila

Zastrow, Leonhard 